\title{
On Fibonacci functions with Fibonacci numbers
}

Jeong Soon Han ${ }^{1}$, Hee Sik Kim² ${ }^{2}$ and Joseph Neggers ${ }^{3}$

${ }^{*}$ Correspondence:

heekim@hanyang.ac.kr

2Department of Mathematics,

Research Institute for Natural

Sciences, Hanyang University, Seoul,

133-791, Korea

Full list of author information is

available at the end of the article

\begin{abstract}
In this paper we consider Fibonacci functions on the real numbers $\mathbf{R}$, i.e., functions $f: \mathbf{R} \rightarrow \mathbf{R}$ such that for all $x \in \mathbf{R}, f(x+2)=f(x+1)+f(x)$. We develop the notion of Fibonacci functions using the concept of $f$-even and $f$-odd functions. Moreover, we show that if $f$ is a Fibonacci function then $\lim _{x \rightarrow \infty} \frac{f(x+1)}{f(x)}=\frac{1+\sqrt{5}}{2}$.

MSC: $11 \mathrm{~B} 39 ; 39 \mathrm{~A} 10$
\end{abstract}

Keywords: Fibonacci function; $f$-even ( $f$-odd) function; Golden ratio

\section{Introduction}

Fibonacci numbers have been studied in many different forms for centuries and the literature on the subject is, consequently, incredibly vast. One of the amazing qualities of these numbers is the variety of mathematical models where they play some sort of role and where their properties are of importance in elucidating the ability of the model under discussion to explain whatever implications are inherent in it. The fact that the ratio of successive Fibonacci numbers approaches the Golden ratio (section) rather quickly as they go to infinity probably has a good deal to do with the observation made in the previous sentence. Surveys and connections of the type just mentioned are provided in [1] and [2] for a very minimal set of examples of such texts, while in [6] an application (observation) concerns itself with a theory of a particular class of means which has apparently not been studied in the fashion done there by two of the authors of the present paper. Recently, Hyers-Ulam stability of Fibonacci functional equation was studied in [5]. Surprisingly novel perspectives are still available and will presumably continue to be so for the future as long as mathematical investigations continue to be made. In the following, the authors of the present paper are making another small offering at the same spot many previous contributors have visited in both recent and more distance pasts. The present authors [3, 4] studied a Fibonacci norm of positive integers and Fibonacci sequences in groupoids in arbitrary groupoids.

In this paper we consider Fibonacci functions on the real numbers $\mathbf{R}, i . e$, functions $f$ : $\mathbf{R} \rightarrow \mathbf{R}$ such that for all $x \in \mathbf{R}, f(x+2)=f(x+1)+f(x)$. We develop the notion of Fibonacci functions using the concept of $f$-even and $f$-odd functions. Moreover, we show that if $f$ is a Fibonacci function then $\lim _{x \rightarrow \infty} \frac{f(x+1)}{f(x)}=\frac{1+\sqrt{5}}{2}$.

(c) 2012 Han et al.; licensee Springer. This is an Open Access article distributed under the terms of the Creative Commons Attribution License (http://creativecommons.org/licenses/by/2.0), which permits unrestricted use, distribution, and reproduction in any medium, provided the original work is properly cited. 


\section{Fibonacci functions}

A function $f$ defined on the real numbers is said to be a Fibonacci function if it satisfies the formula

$$
f(x+2)=f(x+1)+f(x)
$$

for any $x \in \mathbf{R}$, where $\mathbf{R}$ (as usual) is the set of real numbers.

Example 2.1 Let $f(x):=a^{x}$ be a Fibonacci function on $\mathbf{R}$ where $a>0$. Then $a^{x} a^{2}=f(x+$ $2)=f(x+1)+f(x)=a^{x}(a+1)$. Since $a>0$, we have $a^{2}=a+1$ and $a=\frac{1+\sqrt{5}}{2}$. Hence $f(x)=$ $\left(\frac{1+\sqrt{5}}{2}\right)^{x}$ is a Fibonacci function, and the unique Fibonacci function of this type on $\mathbf{R}$.

If we let $u_{0}=0, u_{1}=1$, then we consider the full Fibonacci sequence: ..., $5,-3,2,-1,1,0$, $1,1,2,3,5, \ldots$, i.e., $u_{-n}=(-1)^{n+1} u_{n}$ for $n>0$, and $u_{n}=F_{n}$, the $n$th Fibonacci number.

Example 2.2 Let $\left\{u_{n}\right\}_{n=-\infty}^{\infty}$ and $\left\{v_{n}\right\}_{n=-\infty}^{\infty}$ be full Fibonacci sequences. We define a function $f(x)$ by $f(x):=u_{\lfloor x\rfloor}+v_{\lfloor x\rfloor} t$, where $t=x-\lfloor x\rfloor \in(0,1)$. Then $f(x+2)=u_{\lfloor x+2\rfloor}+v_{\lfloor x+2\rfloor} t=$ $u_{(\lfloor x\rfloor+2)}+v_{(\lfloor x\rfloor+2)} t=\left(u_{(\lfloor x\rfloor+1)}+u_{\lfloor x\rfloor}\right)+\left(v_{(\lfloor x\rfloor+1)}+v_{\lfloor x\rfloor}\right) t=f(x+1)+f(x)$ for any $x \in \mathbf{R}$. This proves that $f$ is a Fibonacci function.

Note that if a Fibonacci function is differentiable on $\mathbf{R}$, then its derivative is also a Fibonacci function.

Proposition 2.3 Let $f$ be a Fibonacci function. If we define $g(x):=f(x+t)$ where $t \in \mathbf{R}$ for any $x \in \mathbf{R}$, then $g$ is also a Fibonacci function.

Proof Given $x \in \mathbf{R}$, we have $g(x+2)=f(x+2+t)=f(x+t+1)+f(x+t)=g(x+1)+g(x)$, proving the proposition.

For example, since $f(x)=\left(\frac{1+\sqrt{5}}{2}\right)^{x}$ is a Fibonacci function, $g(x)=\left(\frac{1+\sqrt{5}}{2}\right)^{x+t}=\left(\frac{1+\sqrt{5}}{2}\right)^{t} f(x)$ is also a Fibonacci function where $t \in \mathbf{R}$.

Example 2.4 In Example 2.2, we discussed the function $f(x):=u_{\lfloor x\rfloor}+v_{\lfloor x\rfloor} t$, where $t=$ $x-\lfloor x\rfloor \in(0,1)$. If we let $v_{\lfloor x\rfloor}:=u_{(\lfloor x\rfloor-1)}$, then $f(x)$ is a Fibonacci function. We compute $f(-6.1)$ and $f(-5.9)$ as follows: $f(-6.1)=f(-7+0.9)=u_{-7}+u_{-8}(0.9)=-5.9$ and $f(-5.9)=$ $f(-6+0.1)=u_{-6}+u_{-7}(0.1)=-6.7$.

Theorem 2.5 Let $f(x)$ be a Fibonacci function and let $\left\{F_{n}\right\}$ be a sequence of Fibonacci numbers with $F_{0}=0, F_{1}=F_{2}=1$. Then $f(x+n)=F_{n} f(x+1)+F_{n-1} f(x)$ for any $x \in \mathbf{R}$ and $n \geq 2$ an integer.

Proof If $n=2$, then $f(x+2)=f(x+1)+f(x)=F_{2} f(x+1)+F_{1} f(x)$. If $n=3$, then we have

$$
\begin{aligned}
f(x+3) & =f(x+2)+f(x+1) \\
& =F_{2} f(x+1)+F_{1} f(x)+F_{1} f(x+1)+F_{0} f(x) \\
& =\left(F_{1}+F_{2}\right) f(x+1)+\left(F_{1}+F_{0}\right) f(x) \\
& =F_{3} f(x+1)+F_{2} f(x) .
\end{aligned}
$$


If we assume that it holds for the cases of $n$ and $n+1$, then

$$
\begin{aligned}
f(x+n+2) & =f(x+n+1)+f(x+n) \\
& =F_{n+1} f(x+1)+F_{n} f(x)+F_{n} f(x+1)+F_{n-1} f(x) \\
& =\left(F_{n+1}+F_{n}\right) f(x+1)+\left(F_{n}+F_{n-1}\right) f(x) \\
& =F_{n+2} f(x+1)+F_{n+1} f(x),
\end{aligned}
$$

proving the theorem.

Corollary 2.6 If $\left\{F_{n}\right\}$ is the sequence of Fibonacci numbers with $F_{1}=F_{2}=1$, then

$$
\left(\frac{1+\sqrt{5}}{2}\right)^{n}=F_{n}\left(\frac{1+\sqrt{5}}{2}\right)+F_{n-1} \text {. }
$$

Proof As we have seen in Example 2.1, $f(x)=\left(\frac{1+\sqrt{5}}{2}\right)^{x}$ is a Fibonacci function. Let $a:=\frac{1+\sqrt{5}}{2}$. By applying Theorem 2.5, we have $a^{x+n}=f(x+n)=F_{n} f(x+1)+F_{n-1} f(x)=F_{n} a^{x+1}+F_{n-1} a^{x}$, proving that $a^{n}=F_{n} a+F_{n-1}$.

Theorem 2.7 Let $\left\{u_{n}\right\}$ be the full Fibonacci sequence. Then

$$
u_{\lfloor x+n\rfloor}=F_{n} u_{(\lfloor x\rfloor+1)}+F_{n-1} u_{\lfloor x\rfloor}
$$

and

$$
u_{(\lfloor x+n\rfloor-1)}=F_{n} u_{\lfloor x\rfloor}+F_{n-1} u_{(\lfloor x\rfloor-1)} .
$$

Proof The map $f(x):=u_{\lfloor x\rfloor}+u_{(\lfloor x\rfloor-1)} t$ discussed in Example 2.4 is a Fibonacci function. If we apply Theorem 2.5 , then we obtain

$$
\begin{aligned}
u_{\lfloor x+n\rfloor}+u_{(\lfloor x+n\rfloor-1)} t & =f(x+n) \\
& =F_{n} f(x+1)+F_{n-1} f(x) \\
& =F_{n}\left[u_{\lfloor x+1\rfloor}+u_{(\lfloor x+1\rfloor-1)} t\right]+F_{n-1}\left[u_{\lfloor x\rfloor}+u_{(\lfloor x\rfloor-1)} t\right] \\
& =F_{n}\left[u_{(\lfloor x\rfloor+1)}+u_{\lfloor x\rfloor} t\right]+F_{n-1}\left[u_{\lfloor x\rfloor}+u_{(\lfloor x\rfloor-1)} t\right] \\
& =\left[F_{n} u_{(\lfloor x\rfloor+1)}+F_{n-1} u_{\lfloor x\rfloor}\right]+\left[F_{n} u_{\lfloor x\rfloor}+F_{n-1} u_{(\lfloor x\rfloor-1)}\right] t,
\end{aligned}
$$

proving the theorem.

Corollary 2.8 If $n \geq 2$, then

$$
F_{\lfloor x+n\rfloor}=F_{n} F_{(\lfloor x\rfloor+1)}+F_{n-1} F_{\lfloor x\rfloor}
$$

and

$$
F_{(\lfloor x+n\rfloor-1)}=F_{n} F_{\lfloor x\rfloor}+F_{n-1} F_{(\lfloor x\rfloor-1)} .
$$

Corollary 2.9 $F_{n+2}=F_{n} F_{3}+F_{n-1} F_{2}$. 
Proof Let $x:=2$ in $(2.5)$ or $x:=3$ in (2.6).

\section{$3 f$-even and $f$-odd functions}

In this section, we develop the notion of Fibonacci functions using the concept of $f$-even and $f$-odd functions.

Definition 3.1 Let $a(x)$ be a real-valued function of a real variable such that if $a(x) h(x) \equiv 0$ and $h(x)$ is continuous then $h(x) \equiv 0$. The map $a(x)$ is said to be an $f$-even function (resp., $f$-odd function) if $a(x+1)=a(x)$ (resp., $a(x+1)=-a(x))$ for any $x \in \mathbf{R}$.

Example 3.2 If $a(x)=x-\lfloor x\rfloor$, then $a(x) h(x) \equiv 0$ implies $h(x)=0$ if $x \notin Z$. By continuity of $h(x)$, it follows that $h(n)=\lim _{x \rightarrow n} h(x)=0$ for any integer $n$, and hence $h(x) \equiv 0$. Since $a(x+1)=(x+1)-\lfloor x+1\rfloor=(x+1)-(\lfloor x\rfloor+1)=x-\lfloor x\rfloor=a(x)$, we see that $a(x)$ is an $f$-even function.

Example 3.3 If $a(x)=\sin (\pi x)$, then $a(x) h(x) \equiv 0$ implies $h(x)=0$ if $x \neq n \pi$ for any integer $n$. By continuity of $h(x)$ it follows that $h(n \pi)=\lim _{x \rightarrow n \pi} h(x)=0$ for any integer $n$, and hence $h(x) \equiv 0$. Since $a(x+1)=\sin (\pi x+\pi)=\sin (\pi x) \cos (\pi)=-\sin (\pi x)=-a(x)$, we see that $a(x)$ is an $f$-odd function.

Theorem 3.4 Let $f(x)=a(x) g(x)$ be a function, where $a(x)$ is an $f$-even function and $g(x)$ is a continuous function. Then $f(x)$ is a Fibonaccifunction if and only if $g(x)$ is a Fibonacci function.

Proof Suppose that $f(x)$ is a Fibonacci function. Then $a(x) g(x+2)=a(x+2) g(x+2)=$ $f(x+2)=f(x+1)+f(x)=a(x)[g(x+1)+g(x)]$. Hence $a(x)[g(x+2)-g(x+1)-g(x)] \equiv 0$ and $g(x+2)-g(x+1)-g(x) \equiv 0$, i.e., $g(x+2)=g(x+1)+g(x)$ and $g(x)$ is a Fibonacci function. On the other hand, if $g(x)$ is any Fibonacci function, then $a(x+2)=a(x+1)+a(x)$ implies that $f(x)=a(x) g(x)$ is also a Fibonacci function.

Example 3.5 It follows from Example 2.1 that $g(x)=\left(\frac{1+\sqrt{5}}{2}\right)^{x}$ is a Fibonacci function. Since $a(x)=x-\lfloor x\rfloor$ is an $f$-even function, by Theorem 3.4, $f(x)=a(x) g(x)=(x-\lfloor x\rfloor)\left(\frac{1+\sqrt{5}}{2}\right)^{x}$ is a Fibonacci function.

Example 3.6 If we define $a(x)=1$ if $x$ is rational and $a(x)=-1$ if $x$ is irrational, then $a(x+1)=a(x)$ for any $x \in \mathbf{R}$. Also, if $a(x) h(x) \equiv 0$, then $h(x) \equiv 0$ whether or not $h(x)$ is continuous. Thus $a(x)$ is an $f$-even function. In Example 3.5, we have seen that $f(x)=$ $(x-\lfloor x\rfloor)\left(\frac{1+\sqrt{5}}{2}\right)^{x}$ is a Fibonacci function. By applying Theorem 3.4, the map defined by

$$
a(x) f(x)= \begin{cases}(x-\lfloor x\rfloor)\left(\frac{1+\sqrt{5}}{2}\right)^{x} & \text { if } x \in Q, \\ -(x-\lfloor x\rfloor)\left(\frac{1+\sqrt{5}}{2}\right)^{x} & \text { otherwise }\end{cases}
$$

is also a Fibonacci function.

Now, we discuss $f$-odd functions with Fibonacci functions. Let $a(x)$ be an $f$-odd function and $g(x)$ be a continuous function. Let $f(x)$ be a Fibonacci function such that $f(x)=$ 
$a(x) g(x)$. Then $a(x)[g(x+2)+g(x+1)-g(x)]=0$. In this situation, the characteristic equation $r^{2}+r-1=0$ yields solutions of the type $\frac{-1 \pm \sqrt{5}}{2}$, and thus for $r>0$, the solution type is $g(x)=\left(\frac{\sqrt{5}-1}{2}\right)^{x}$, whereas $\left(\frac{-1-\sqrt{5}}{2}\right)^{x}$ is not a real number except for special values of $x$.

A function $f$ defined on $\mathbf{R}$ satisfying $f(x+2)=-f(x+1)+f(x)$ for all $x \in \mathbf{R}$ is said to be an odd Fibonacci function. Similarly, a sequence $\left\{a_{n}\right\}_{n=0}^{\infty}$ with $a_{n+2}=-a_{n+1}+a_{n}$ is said to be an odd Fibonacci sequence.

Example 3.7 A sequence $\{1,1,0,1,-1,2,-3,5, \ldots\}$ is an odd Fibonacci sequence.

Corollary 3.8 Let $f(x)=a(x) g(x)$ be a function, where $a(x)$ is an $f$-odd function and $g(x)$ is a continuous function. Then $f(x)$ is a Fibonacci function if and only if $g(x)$ is an odd Fibonacci function.

Proof Similar to the proof of Theorem 3.4.

Example 3.9 The function $g(x)=\left(\frac{\sqrt{5}-1}{2}\right)^{x}$ is an odd Fibonacci function. Since $a(x)=$ $\sin (\pi x)$ is an $f$-odd function, by Corollary 3.8, we can see that the function $f(x)=$ $\sin (\pi x)\left(\frac{\sqrt{5}-1}{2}\right)^{x}$ is a Fibonacci function.

\section{Quotients of Fibonacci functions}

In this section, we discuss the limit of the quotient of a Fibonacci function.

Theorem 4.1 If $f(x)$ is a Fibonacci function, then the limit of quotient $\frac{f(x+1)}{f(x)}$ exists.

Proof If we consider a quotient $\frac{f(x+1)}{f(x)}$ of a Fibonacci function $f(x)$, we have 4 cases: (i) $f(x)>0, f(x+1)>0$; (ii) $f(x)<0, f(x+1)>0$; (iii) $f(x)>0, f(x+1)<0$; (iv) $f(x)<0$, $f(x+1)<0$. Consider (iii). If we let $\alpha:=f(x)>0, \beta:=f(x+1)<0$, then $f(x+2)=\alpha-\beta$, $f(x+3)=\alpha-2 \beta, f(x+4)=2 \alpha-3 \beta=F_{3} \alpha-F_{4} \beta$ and $f(x+5)=F_{4} \alpha-F_{5} \beta$. In this fashion, we obtain $f(x+n)=F_{n} \alpha-F_{n+1} \beta$ for any natural number $n \in \mathbf{N}$. Given $x^{\prime} \in \mathbf{R}$, there exist $x \in \mathbf{R}$ and $n \in \mathbf{Z}$ such that $x^{\prime}=x+n$. Hence

$$
\begin{aligned}
\frac{f\left(x^{\prime}+1\right)}{f\left(x^{\prime}\right)} & =\frac{f(x+n+1)}{f(x+n)} \\
& =\frac{F_{n+1} \alpha-F_{n} \beta}{F_{n} \alpha-F_{n-1} \beta} \\
& =\frac{\frac{F_{n+1}}{F_{n}} \alpha-\beta}{\alpha-\frac{F_{n}}{F_{n-1}}} \\
& \rightarrow \frac{\Phi \alpha-\beta}{\alpha-\frac{\beta}{\Phi}}=\Phi,
\end{aligned}
$$

where $\lim _{n \rightarrow \infty} \frac{F_{n+1}}{F_{n}}=\Phi=\frac{1+\sqrt{5}}{2}$. Thus $\lim _{x \rightarrow \infty} \frac{f(x+1)}{f(x)}=\Phi$. Case (ii) is similar to the case (iii). Consider the case (i): $f(x)>0, f(x+1)>0$. We may change $\frac{f(x+1)}{f(x)}$ by $\frac{f(\delta+2 n+1)}{f(\delta+2 n)}$, since any real number $x(>0)$ can be written $x=\delta+2 n$ for some $\delta \in \mathbf{R}$ and $n \in \mathbf{N}$. Consider a sequence $\left\{\frac{f(\delta+2 n+1)}{f(\delta+2 n)}\right\}_{n=1}^{\infty}$.

$$
\frac{f(\delta+2 n+1)}{f(\delta+2 n)}=\frac{f(\delta+2 n)+f(\delta+2 n-1)}{f(\delta+2 n)}=1+\frac{f(\delta+2 n-1)}{f(\delta+2 n)}<2,
$$


since $\frac{f(\delta+2 n-1)}{f(\delta+2 n)}<1$. We claim that $\left\{\frac{f(\delta+2 n+1)}{f(\delta+2 n)}\right\}_{n=1}^{\infty}$ is monotonically increasing. Since $\frac{f(\delta+2 n+3)}{f(\delta+2 n+2)}-$ $\frac{f(\delta+2 n+1)}{f(\delta+2 n)}=\frac{f(\delta+2 n+3) f(\delta+2 n)-f(\delta+2 n+2) f(\delta+2 n+1)}{f(\delta+2 n+2) f(\delta+2 n)}$, we show that the numerator part of the quotient is positive.

$$
\begin{aligned}
f(\delta & +2 n+3) f(\delta+2 n)-f(\delta+2 n+2) f(\delta+2 n+1) \\
& =[f(\delta+2 n+2)+f(\delta+2 n+1)] f(\delta+2 n)-f(\delta+2 n+2) f(\delta+2 n+1) \\
& =f(\delta+2 n+2)[f(\delta+2 n)-f(\delta+2 n+1)]+f(\delta+2 n+1) f(\delta+2 n) \\
& >f(\delta+2 n)[f(\delta+2 n)-f(\delta+2 n+1)]+f(\delta+2 n+1) f(\delta+2 n) \\
& =[f(\delta+2 n)]^{2} \\
& \geq 0,
\end{aligned}
$$

which shows that the sequence is monotonically increasing. By the Monotone Convergence Theorem, there exists $\lim _{x \rightarrow \infty} \frac{f(\delta+2 n+1)}{f(\delta+2 n)}=\lim _{x \rightarrow \infty} \frac{f(x+1)}{f(x)}$. The case (iv) is similar to the case (i). This proves the theorem.

\section{Corollary 4.2 Iff $(x)$ is a Fibonacci function, then}

$$
\lim _{x \rightarrow \infty} \frac{f(x+1)}{f(x)}=\frac{1+\sqrt{5}}{2} .
$$

Proof If we let $\alpha:=f(x)>0, \beta:=f(x+1)>0$, then

$$
\begin{aligned}
\frac{f(x+n+1)}{f(x+n)} & =\frac{F_{n+1} \alpha+F_{n+2} \beta}{F_{n} \alpha+F_{n+1} \beta} \\
& =\alpha+\frac{\frac{F_{n+2}}{F_{n+1}} \beta}{\frac{F_{n}}{F_{n+1}}+\beta} \\
& \rightarrow \frac{\alpha+\Phi \beta}{\frac{\alpha}{\Phi}+\beta}=\Phi .
\end{aligned}
$$

It is shown already in the proof of Theorem 4.1 for the case of $\alpha:=f(x)>0, \beta:=f(x+1)<0$ that the limit of the quotient $\frac{f(x+n+1)}{f(x+n)}$ converges to $\Phi$, proving the corollary.

\section{Competing interests}

The authors declare that they have no competing interests.

\section{Authors' contributions}

All authors conceived of the study, participated in its design and coordination, drafted the manuscript, participated in the sequence alignment, and read and approved the final manuscript.

\section{Author details}

'Department of Applied Mathematics, Hanyang University, Ahnsan, 426-791, Korea. ${ }^{2}$ Department of Mathematics, Research Institute for Natural Sciences, Hanyang University, Seoul, 133-791, Korea. ${ }^{3}$ Department of Mathematics, University of Alabama, Tuscaloosa, AL 35487-0350, USA.

\section{Acknowledgement}

The authors are grateful to the referee's valuable suggestions and help. 


\section{References}

1. Atanasov, K, et al.: New Visual Perspectives on Fibonacci Numbers. World Scientific, Hackensack (2002).

2. Dunlap, RA: The Golden Ratio and Fibonacci Numbers. World Scientific, Hackensack (1997)

3. Han, JS, Kim, HS, Neggers, J: The Fibonacci norm of a positive integer n-observations and conjectures. Int. J. Number Theory 6, 371-385 (2010)

4. Han, JS, Kim, HS, Neggers, J: Fibonacci sequences in groupoids. Adv. Differ. Equ. 2012, 19 (2012). doi:10.1186-11847-2012-19

5. Jung, SM: Hyers-Ulam stability of Fibonacci functional equation. Bull. Iran. Math. Soc. 35, 217-227 (2009)

6. Kim, HS, Neggers, J: Fibonacci means and golden section mean. Comput. Math. Appl. 56, 228-232 (2008)

doi:10.1186/1687-1847-2012-126

Cite this article as: Han et al.: On Fibonacci functions with Fibonacci numbers. Advances in Difference Equations 2012 2012:126.

Submit your manuscript to a SpringerOpen ${ }^{\circ}$ journal and benefit from:

- Convenient online submission

- Rigorous peer review

- Immediate publication on acceptance

Open access: articles freely available online

- High visibility within the field

- Retaining the copyright to your article 